\title{
OrganolD: a versatile deep learning platform for organoid image analysis
}

Jonathan Matthews ${ }^{\dagger, 1,2,3}$, Brooke Schuster ${ }^{\dagger, 1,2,4}$, Sara Saheb Kashaf ${ }^{1,2,3}$, Ping Liu, ${ }^{5}$ Mustafa Bilgic ${ }^{5}$, Andrey

Rzhetsky ${ }^{2,6}$, and Savaş Tay ${ }^{1,2^{*}}$

${ }^{1}$ Pritzker School of Molecular Engineering, The University of Chicago, Chicago, IL, 60637, USA;

2 Institute for Genomics and Systems Biology, The University of Chicago, Chicago, IL, 60637, USA;

${ }^{3}$ The University of Chicago Pritzker School of Medicine, Chicago, IL, 60637, USA;

${ }^{4}$ Department of Chemistry, The University of Chicago, Chicago, IL, 60637, USA;

${ }^{5}$ Department of Computer Science, Illinois Institute of Technology, Chicago, IL, 60616, USA;

${ }^{6}$ Committee on Genetics, Genomics and Systems Biology, Departments of Medicine and Human Genetics, The

University of Chicago, Chicago, IL, 60637, USA;

*Correspondence:tays@uchicago.edu

t These authors contributed equally to this work.

\section{ABSTRACT}

Organoids are three-dimensional in vitro tissue models that closely represent the native heterogeneity, microanatomy, and functionality of an organ or diseased tissue. Analysis of organoid morphology, growth, and drug response is challenging due to the diversity in shape and size of organoids, movement through focal planes, and limited options for live-cell staining. Here, we present OrganoID, an opensource image analysis platform that automatically recognizes, labels, and tracks single organoids in brightfield and phase-contrast microscopy. The platform identifies organoid morphology pixel by pixel without the need for fluorescence or transgenic labeling and accurately analyzes a wide range of organoid types in time-lapse microscopy experiments. OrganolD uses a modified u-net neural network with minimal feature depth to encourage model generalization and allow fast execution. The network was trained on images of human pancreatic cancer organoids and was validated on images from pancreatic, lung, colon, and adenoid cystic carcinoma organoids with a mean intersection-over-union of 0.76. OrganolD measurements of organoid count and individual area concurred with manual measurements at $96 \%$ and $95 \%$ agreement respectively. Tracking accuracy remained above $89 \%$ over the duration of a four-day validation experiment. Automated single-organoid morphology analysis of a dose-response experiment identified significantly different organoid circularity after exposure to different concentrations of gemcitabine. The OrganolD platform enables straightforward, detailed, and accurate analysis of organoid images to accelerate the use of organoids as physiologically relevant models in high-throughput research. 


\section{INTRODUCTION}

Three-dimensional (3D) cell culture systems serve as more physiologically relevant in vitro models than traditional human cell monolayers for both basic and clinical applications ${ }^{1}$. Organoids are multicellular 3D structures derived from primary or stem cells that are embedded into a biological matrix to create an extracellular environment that provides structural support and key growth factors. Organoids are a particularly useful culture system, as they closely recapitulate cellular heterogeneity, structural morphology, and organ-specific functions of a variety of tissues ${ }^{2-5}$. Live-cell imaging can reveal important organoid dynamics, such as growth, apoptosis/necrosis, and movement, which can reflect physiological and pathological behaviors and drug responses. While organoids have been successfully used to investigate important phenomena that might be obscured in simpler models, their use in data-intensive applications, such as high-throughput screening, has been limited.

A major challenge for high-throughput organoid experiments is response measurement and data analysis, which must be performed for a large number of microscopy images. Image analysis is particularly difficult for organoid experiments due to their movement across focal planes and variability in organoid size and shape between different tissue types, within the same tissue type, and within the same single culture sample ${ }^{6,7}$. Cells can be genetically modified to express fluorescent proteins ${ }^{8-10}$ that aid in image segmentation and tracking, yet the transformation process increases experimental time and complexity and may alter cellular dynamics from original samples ${ }^{11,12}$. Small fluorescent dyes can penetrate live cell membranes to stain intracellular structures, however this approach is inadequate for organoid culture due to cumulative toxicity over longer growth times and limited diffusion through the hydrogel matrix ${ }^{13}$. There is a critical need for an automated image analysis tool that can measure detailed organoid responses without the use of potentially toxic or confounding live-cell fluorescence techniques.

A number of software tools have been developed to automate the process of organoid image analysis. These platforms use conventional image processing methods, such as adaptive thresholding and mathematical morphology ${ }^{14}$, or convolutional neural networks ${ }^{15,16}$ to identify organoids in sequences of microscopy images. Despite their advantages, most existing platforms require cellular nuclei to be transgenically labeled ${ }^{16}$, which increases experiment time and complexity and may modify cellular dynamics, require manual parameter tuning for each image ${ }^{14}$, or are limited to bounding-box detection ${ }^{15}$, which fails to capture potentially useful morphological information. Changes in organoid shape, such as spiking or blebbing, can reveal important responses to external stimuli organoids and might be missed with bounding-box measurements ${ }^{30}$. Importantly, existing platforms were developed for analysis of organoids derived from a single type of tissue and for images obtained with one specific geometric configuration.

To address these challenges, we have developed a software platform, OrganoID, that can identify and track organoids derived from a wide range of tissue types, pixel by pixel, in both brightfield and phase-contrast microscopy images. OrganolD consists of (i) a convolutional neural network, which detects organoids in microscopy images, (ii) an identification module, which resolves contours to label individual organoids, and (iii) a tracking module, which follows identified organoids in time-lapse imaging experiments. Most importantly, OrganoID accurately segments and tracks a wide range of organoid types, including those derived from 
pancreatic ductal adenocarcinoma, adenoid cystic carcinoma, and lung and colon epithelia. OrganoID is publicly available both as source code and a standalone executable. The distribution package also includes sample datasets and detailed usage instructions and examples. The OrganoID software overcomes a major hurdle to organoid image analysis and supports wider integration of the organoid model into high-throughput applications.

\section{RESULTS}

\section{A robust convolutional neural network for pixel-by-pixel organoid detection}

We developed a deep learning-based image analysis pipeline, OrganoID, that recognizes and tracks individual organoids, pixel by pixel, for bulk and single-organoid analysis in brightfield and phase-contrast microscopy images (Figure 1a). The platform employs a convolutional neural network to transform microscopy images into organoid probability images, where brightness values represent the network belief that an organoid is present at a given pixel. The network structure was derived from the widely successful u-net approach to image segmentation ${ }^{9}$ (Figure 1b). The u-net approach first passes each image through a contracting series of multidimensional convolutions and maximum filters to extract a set of deep feature maps that describe the image at various levels of detail and contexts, such as edges, shapes, and textures. The feature maps are then passed through an expanding series of transposed convolutions, which learn to localize the features and assemble a final output. The OrganoID neural network follows a $u$-net structure and was optimized to require far fewer feature channels than the original implementation. Model simplification can limit overfitting and minimizes the amount of memory and computational power required to use the network in the final distribution. Additionally, all hidden convolutional layers were set to compute outputs with the exponential linear unit (ELU) activation function ${ }^{18}$, which has demonstrated higher accuracy than the rectified linear unit (ReLU) and avoids vanishing gradient problems during network training. Neurons in the final convolutional layer were set with a sigmoid activation function to produce a normalized output that corresponds to the probability that an organoid is present at each pixel in the original image.

An original dataset of 66 brightfield and phase-contrast microscopy images of organoids were manually segmented to produce black-and-white ground truth images for network training and validation (Figure 1c). Each image featured 5 to 50 organoids derived from human pancreatic ductal adenocarcinoma (PDAC) samples from two different patients. Organoids were either grown on a standard tissue culture plate or on our previously published microfluidic organoid platform ${ }^{19}$. To teach the network that segmentations should be independent of imaging orientation, field-of-view, lens distortion, and other potential sources of overfitting, the training dataset was augmented with random rotation, zoom, elastic distortion, and shear transformations to produce a total of 2,000 images (Figure 1d).

Network training halted after 37 epochs, once segmentation error (binary cross-entropy) on the validation dataset converged to a minimal value (Supp. Figure 1, Video 1). After hyperparameter tuning, the final model performance was assessed on a novel PDAC testing dataset, previously unseen by the network. Performance was quantified with the intersection-over-union (IOU) metric, which is defined as the overlap between the predicted and actual organoid pixels divided by their union (Figure 2a). An IOU greater than 0.5 is generally 
considered to reflect a good prediction and we chose this value to be the minimal benchmark for satisfactory model performance. All PDAC images passed the benchmark, with a mean IOU of 0.74 (SD = 0.081) (Figure 2b). Because the network was trained and assessed solely with images of PDAC organoids, we were also curious to evaluate its capacity to generalize to organoids derived from non-PDAC tissues. 18 microscopy images of organoids derived from lung epithelia, colon epithelia, and salivary adenoid cystic carcinoma (ACC) were manually segmented. The OrganoID network passed the benchmark for all non-PDAC images, with a mean IOU of 0.79 (SD=0.096). These results demonstrate the capability of the OrganolD neural network to reliably detect organoids from various tissues of origin in a pixel-by-pixel manner (Figure 2c).

The network was also evaluated for appropriate exclusion of non-organoid technical artifacts. Air bubbles in culture media or gel matrix were rarely detected by OrganoID with a false positive rate of $4.2 \%$ (Supp. Figure 2a). We observed that OrganolD segmentations also avoided cellular debris or dust embedded into the gel, ignored chamber borders, and performed robustly across microscope resolutions, organoid concentrations, and organoid shapes (Supp. Figure $\mathbf{2 b - g}$ ). Each image was segmented in 300 milliseconds on a laptop CPU (Intel $\mathrm{i} 7-9750 \mathrm{H}, 2.6 \mathrm{GHz}$ ) with less than 200 megabytes of RAM usage.

\section{Identification and tracking of individual organoids with diverse morphology and size}

The convolutional neural network detects organoids in an image on a pixel-by-pixel basis, which can be used to measure bulk responses. For single-organoid analysis, pixels must be grouped together to identify individual organoids. This task is straightforward for isolated organoids, where all high-belief pixels in a cluster correspond to one organoid, but is more difficult for organoids that are in physical contact. To address this challenge, we developed an organoid separation pipeline (Figure 3a) that uses the raw network prediction map to group pixels into single-organoid clusters. Conventionally, neural network image segmentation methods set an absolute threshold on predicted pixels to produce a binary detection mask. This approach is effective, but discards useful information about the strength of predictions. The segmentations that were used to train the neural network were produced with a thin boundary between organoids in contact. As a result, the network predictions were marginally less confident about pixels near organoid boundaries. We took advantage of this phenomenon to identify and separate organoid contours with a modified Canny edge detector ${ }^{20}$ and a watershed transformation. Our detector sequentially applies (i) a pair of Sobel operators, which compute the image intensity gradient; (ii) a Gaussian filter, which smooths noisy regions; and (iii) a hysteresis-based threshold, which identifies locally strong edges. Edges are removed from the thresholded prediction image to mark the centers of each organoid. These centers are then used as initializer basins for a watershed transformation applied to the raw prediction image. The image is further refined to remove organoids that may be partially out of the field-of-view or are below a particular size threshold. The pipeline outputs a labeled image, where the pixels that represent an individual organoid are all set to a unique organoid ID number, which can be used for single-organoid analysis (Figure 3a).

For quantitative validation, OrganoID was used to count and measure the area of organoids in images from the PDAC and non-PDAC testing datasets (a total of 28 images). These data were then compared to the number and area of organoids in the corresponding manually segmented images. Organoid counts agreed with 
a concordance correlation coefficient (CCC) of 0.96 [95\% Cl 0.91-0.98]. OrganolD, on average, detected 0.5 fewer organoids per image than manual segmentation. The limits of agreement between OrganoID and manual counts were between -8.5 and 7.5 organoids (Figure $\mathbf{3 b}$ ). Organoid area comparison demonstrated a CCC of 0.95 [95\% Cl 0.94-0.96]. OrganoID area measurements were biased to be $0.91 \times 10^{3} \mu^{2}$ larger per organoid with limits of agreement between $-5.47 \times 10^{3} \mu \mathrm{m}^{2}$ and $7.28 \times 10^{3} \mu \mathrm{m}^{2}$ (Figure 3c). Overall, the measurements produced by OrganolD were in considerable agreement with those obtained by hand, which supports the use of OrganolD for automated single-organoid analysis.

OrganoID was also developed with a tracking module for longitudinal single-organoid analysis of timecourse imaging experiments, where changes in various properties, such as size and shape, of individual organoids can be measured and followed over time. The central challenge for the tracking module is to match a detected organoid in a given image to the same organoid in a later image. The Hungarian assignment algorithm ${ }^{21}$ was used to minimize a cost matrix based on the number of shared pixels between detected organoids in two images. This approach produces organoid "tracks" for unique organoids in time-lapse images. For validation, microscopy images taken every 4 hours from a 92-hour organoid culture experiment were passed through the entire OrganoID pipeline to produce growth curves that followed single-organoid changes over time (Figure 3d, e). The tracking step was also performed by hand to evaluate automated performance, which maintained over $89 \%$ accuracy throughout the duration of the experiment (Supp. Figure 3, Video 2).

\section{OrganoID measures bulk and single-organoid death responses over time}

PDAC organoids were treated with serial dilutions of gemcitabine (3nM-1000nM), an FDA-approved chemotherapeutic agent commonly used to treat pancreatic cancer, and imaged every 4 hours of the course of 72 hours. Propidium iodide (PI), a fluorescent reporter of cellular necrosis, was also added to the culture media to monitor death responses. Brightfield images were then processed with the OrganolD platform to identify organoids and analyze bulk and single-organoid responses (Figure 4a). At gemcitabine concentrations above 3 $\mathrm{nM}$, the total organoid area increased for the first several hours, which reflected initial organoid growth, but then decreased to a value and at a rate inversely proportional to gemcitabine concentration (Figure 4b). Identified organoid counts for gemcitabine concentrations above $10 \mathrm{nM}$ also sharply decreased over time. The total fluorescence intensity of the PI signal increased over time to a value and at a rate proportional to gemcitabine concentration, however the response to $100 \mathrm{nM}$ gemcitabine appeared to induce a stronger death signal than the response to $1000 \mathrm{nM}$ gemcitabine (Figure 4c). Measurements of death stains such as $\mathrm{PI}$ are typically normalized to a viability measurement that accounts for differences in the number and size of organoids between replicates that can compound over the duration of an experiment. We used OrganolD to normalize fluorescence measurements: the total fluorescence intensity of identified organoid regions was divided by the total organoid area at each time point. Normalization with OrganoID increased the separation of responses between each treatment group over time, decreased standard error across replicates, and corrected the response discrepancy between the $100 \mathrm{nM}$ and $1000 \mathrm{nM}$ conditions (Figure 4c). We also compared these normalized measurements to normalization with an MTS proliferation assay, which can only be used for endpoint analysis. Normalization 
with OrganolD identified the same significantly different endpoint responses as MTS assay normalization (Supp.

\section{Figure 4, Supp. Table 1).}

Changes in organoid morphology can indicate important phenotypic responses and state transitions ${ }^{30}$. OrganoID was used to identify and profile single organoids in images at the experiment endpoint for gemcitabine concentrations of $100 \mathrm{nM}, 10 \mathrm{nM}$, and the negative control. The fluorescence of individual organoids did not significantly differ across concentrations of gemcitabine (Supp. Figure 5), however fluorescence normalized to single-organoid area was significantly different $(p<0.001)$. Organoid circularity, a morphological measurement that compares the organoid perimeter to the perimeter of a perfect circle, was also significantly different across gemcitabine dosages ( $\mathrm{p}<0.001)$, which supports disruption of organoid architecture by gemcitabine (Figure 4d). This worked example demonstrates the advantages of OrganolD for automated bulk and single-organoid morphological analysis of time-course experiments without the need for live-cell fluorescence techniques.

\section{DISCUSSION}

Organoids have revolutionized biomedical research through improved model representation of native tissues and organ systems. However, the field has yet to fully enter the high-throughput experimental space. A central bottleneck is the challenge of automated response measurement and analysis in large numbers of microscopy images. Organoids exhibit striking diversity in morphology and size and can move through their 3D environment into and out of the focal plane; current image processing tools have not quite been able to capture these aspects in a robust manner. We developed OrganoID to bridge this gap and automate the process of accurate pixel-bypixel organoid identification and tracking.

Experimental replicates in organoid studies can differ in the number and distribution of sizes of organoids. This difference between identical conditions requires per-sample normalization of response measurements to account for differences in baseline growth of organoid colonies. There are several commercially available livecell assays that can facilitate normalization in 2D culture. However, these same assays have proven to be difficult for organoid use due to the production of toxic photobleaching byproducts, limited diffusion through the gel matrix, and nonspecific staining of the gel matrix that results in a considerable background signal. Another available option is to genetically modify each organoid sample to express fluorescent proteins, however this increases experimental time and complexity and may alter cellular dynamics from the original tissue sample. The OrganoID platform can be leveraged for accurate normalization of standard organoid assays without livecell fluorescence methods. OrganolD is also uniquely useful for efficient quantification of single-organoid morphological features, such as circularity, that can reflect important dynamic responses.

Additionally, we have contributed a manually segmented organoid image dataset for use in other computational platforms. OrganoID has demonstrated compatibility with organoids of various sizes, shapes, and sample concentrations as well as various optical configurations. Most excitingly, the OrganolD model was trained and validated on images of PDAC organoids but still demonstrated excellent generalization to images of other types of organoids, including those derived from colon tissue, lung tissue, and adenoid cystic carcinoma. 
OrganolD was trained with and tested on a diverse, but relatively small set of images. Despite the suggested generalizability of OrganoID to various samples and optical configurations, performance may still differ with other types of organoids or microscopes. As well, OrganolD can only detect and assign a single organoid to each pixel in an image. While the platform can appropriately identify contours of organoids in physical contact, it cannot distinguish organoids that overlap across the focal plane. These limitations can be overcome with additional validation, and perhaps an expanded training dataset, as well as the use of multiple focal planes for image analysis.

We have released the OrganolD platform open-source and freely licensed on GitHub. The repository includes all source code and a compiled executable, as well as the entire training and testing dataset, usage instructions, and scripts used for the examples presented in this paper. The network training module is also included on the repository to allow further model training to improve performance for any untested applications.

Acknowledgements: We would like to acknowledge Dr. Dana Ishay-Ronen and Dr. Rakefet Ruth Ben-Yishay from Sheba Medical Center for providing the airway lung organoids; Dr. Sonia Kupfer and Margaret Bielski from University of Chicago Medicine for providing the colon organoids; Dr. Chris Weber and Dr. Le Shen from University of Chicago Medicine for providing the adenoid cystic carcinoma (ACC) organoids.

Author contributions: J.M.M., B.S., S.S.K. and S.T. were involved with the conception of the platform. J.M.M. and B.S. wrote the manuscript, collaborated on platform design, and created the manual organoid segmentation dataset. B.S. performed all organoid experiments and the associated data/statistical analysis and interpretation. S.S.K developed and evaluated the initial deep learning segmentation approach. J.M.M. optimized the neural network, developed the single-organoid pipeline, and implemented the OrganolD software architecture, image processing algorithms, and performance quantification tools. A.R., M.B., S.T. supervised the work and provided technical support. All authors commented on the manuscript.

Competing Interests: The authors declare no competing interests.

Correspondence: Savaş Tay, Institute for Molecular Engineering, The University of Chicago, tays@uchicago.edu

Funding: This work is supported by NIH R01 GM127527 and P. G. Allen Distinguished Investigator Award to S.T. 


\section{METHODS}

\section{Organoid culture and image acquisition}

Tumor organoid cultures derived from pancreatic adenocarcinoma (PDAC) patients were isolated and prepared as previously described ${ }^{22}$. PDAC organoids were cultured and imaged in a microfluidic platform ${ }^{19}$ or a 24 -well suspension culture plate (Thermo Fisher, 144530). Human colonic organoids were cultured similarly to PDAC organoids, with Matrigel and growth media as described in established methods ${ }^{23}$. Distal respiratory organoids were obtained from the Ishay-Ronen Lab at the Sheba Medical Center and cultured through a previously described protocol ${ }^{24}$ in 24-well plates. Images of adenoid cystic carcinoma (ACC) organoids were directly obtained from Dr. Weber and colleagues from University of Chicago Medicine. The pancreatic and airway lung organoids were cultured and imaged on an automated translational stage of an inverted microscope (Nikon Eclipse Ti) enclosed in an environmentally controlled chamber (Life Imaging Service GmbH, Basel, Switzerland). The enclosure provides temperature, humidity, and $\mathrm{CO} 2$ gas control to maintain adequate cell culture conditions for the organoids. Organoids were cultured at a constant $37^{\circ} \mathrm{C}, 5 \% \mathrm{CO}$, a humidity flow rate of $25-30 \mathrm{~L} / \mathrm{hour}$, and $95-100 \%$ relative humidity. Images of the organoids were acquired through the standard microscope software that is capable of automatically acquiring images at different positions, Z-planes/stacks, and multiple fluorescent filters (NIS-Elements software, Japan). The microscope was equipped with a digital complementary metal-oxide semiconductor (CMOS) camera (ORCA-Flash 4.0, Hamamatsu, Japan), which imaged the organoids using a $\times 10$ objective at 2 to 4 -hour intervals.

\section{Neural network design and implementation}

The neural network architecture was based on the u-net encoder-decoder network, which has produced successful results for a variety of biomedical image segmentation tasks ${ }^{9}$. u-net uses several convolutional filters for each layer of neurons and doubles the number of filters per-channel for each sequential layer. The original u-net implementation uses 64 filters in the first layer, which results in quite a large number of trainable parameters across the full network (over 30 million). We sequentially reduced the number of filters in the first layer by powers of two to reach a minimal value that preserved performance on the validation dataset. The final OrganolD $u$-net structure uses only 8 filters in the first layer, which results in a network structure with less than 500,000 trainable parameters (a 98\% reduction compared to the original implementation). This simplification reduces the computational power needed to train and use the network and, in theory, decreases network overfitting. All convolutional neurons were set to compute outputs with the exponential linear unit activation function ${ }^{10}$. The final $1 \times 1$ convolution was set with a sigmoid activation function to produce a normalized output that corresponded to the probability of an organoid at each pixel. All images are auto-contrasted and resized to $512 \times 512$ pixels before training and inference. Python was used for the entire OrganolD platform and Keras (an interface to the TensorFlow library) was used for network expression, training, and operation. The TensorFlow Lite API was used to minimize the memory footprint and number of software dependencies required for network inference in the OrganoID standalone distribution. 


\section{Training procedure and ground-truth dataset creation}

Ground-truth segmentations were created by hand with Pinta and Aseprite, two open-source image editing programs. 66 ground-truth segmentations were created from brightfield and phase-contrast microscopy images of PDAC organoids and were randomly spilt into datasets for training (52 image pairs) and validation (14 image pairs). The Augmentor Python package ${ }^{25}$ was used to apply random rotation, zoom, shear, elastic distortion, and skew operations to the training dataset for data augmentation. The neural network was trained with the Adam stochastic optimization algorithm ${ }^{26}$ at a learning rate of 0.001 . Unweighted binary cross-entropy between predicted and ground-truth segmentations was used for the loss function. Layer weights were initialized with the He method ${ }^{27}$. An early stopping rule was used to halt training once performance on the validation dataset reached a minimum (i.e. once 10 epochs pass with no improvement in validation loss). The batch size was set to train on 8 augmented images for every round of backpropagation. Dropout regularization was introduced after all convolutions to randomly set $12.5 \%$ of neuron weights to zero after each batch. After each epoch, a copy of the model was saved for additional evaluation of the training process.

\section{Dataset for evaluation of network performance and generalizability}

The network structure and training process were manually tuned for performance on the PDAC validation dataset. After optimization, we locked out any further changes to all hyperparameters and evaluated final network performance and generalizability on a separate dataset of 28 organoid microscopy images. The dataset included images of organoids derived from PDAC (10), benign colon tumor cells (6), long epithelia (6), and salivary adenoid cystic carcinoma (6). The images were obtained through brightfield and phase-contrast microscopy from multiple microscopy cores and manually labeled by two independent reviewers.

\section{Single-organoid identification and segmentation refinement}

The scipy ${ }^{28}$ and scikit-image ${ }^{29}$ packages were used to identify individual organoids from the network detection images. Detection images were thresholded at 0.5 and passed through a morphological opening operation to remove weak and noisy predictions. Partial derivatives were computed with a Sobel filter, passed through a Gaussian filter $(\sigma=2)$, and converted to an edge mask with a hysteresis threshold filter $\left(T_{h i}=0.05, T_{10}=0.005\right)$, which was then was used to mark organoid centers. The watershed method was used to identify filled organoid contours, with the organoid centers as label initializers and the network prediction image as an inverted heightmap. Labeled organoids were morphologically filled and discarded if the total area was below 200 pixels. Images were morphologically processed with scikit-image to record properties of identified organoids.

\section{Tracking of individual organoids over time}

The tracking algorithm builds a cost-assignment matrix for each image in a time-lapse sequence, where each row corresponds to an organoid from the previous image and each column corresponds to an organoid in the next image. Each matrix entry is the cost of assigning a given organoid detection in the current image to a detection in the previous image. The cost function was defined as the inverse of the number of shared pixels 
between the two organoids. As such, the assignment cost between two detections will be minimal for those that are of similar shape and location. The cost-assignment matrix is also padded with additional rows and columns to allow for "pseudo-assignments" that represent missing or newly detected organoids. Finally, the Munkres variant of the Hungarian algorithm ${ }^{21}$ was used to minimize the cost-assignment matrix to find an optimal matching between organoid detections in the previous image and the detections in the current image

\section{Statistical methods for platform validation}

Statistical analysis was performed with the numpy and scipy packages in Python. Network performance on the testing dataset was evaluated as the pixel-wise intersection-over-union (IOU) of predictions compared to groundtruth segmentations. A single IOU value was computed for each prediction/ground-truth pair and summarized as a mean IOU and standard deviation over the dataset. For agreement of single-organoid counts and measurements, the Lin concordance correlation coefficient (CCC) was computed as

$$
C C C=\frac{2 s_{x y}}{s_{x}^{2}+s_{y}^{2}+(\bar{x}-\underline{y})^{2}} .
$$

The Fisher transformation ( $\operatorname{artanh}(C C C))$ and the inverse Fisher transformation $\left(\tanh \left(Z_{L O}, Z_{H I}\right)\right)$ were also used to obtain a z-value and construct a 95\% confidence interval for CCC statistics. All plots were generated with the matplotlib and seaborn packages in Python.

\section{Drug screening experiments}

PDAC organoids were grown for one week in standard culture conditions and then treated with gemcitabine hydrochloride (G6423, Sigma) at six serial dilutions from $3 \mathrm{nM}$ to $1000 \mathrm{nM}$ with negative control. Propidium iodide (Thermo Fisher P3566) was used to fluorescently measure cellular death and relative viability of organoids in real-time at 4-hour intervals over 72 hours. An MTS proliferation assay (Promega, G3580) was also performed at the end of the experiment to determine cell viability for each condition according to manufacturer's instructions. Statistical hypothesis testing was performed with scipy and MATLAB. Organoid circularity was computed as the circumference of a circle with equivalent area divided by the actual perimeter of the organoid.

\section{Validation rigor and indications of robustness}

The OrganolD neural network was tested on images of four types of organoids taken at multiple microscopy cores. Images were manually segmented by two independent evaluators. OrganolD single-organoid count and area measurements were compared against the corresponding measurements of the manual test segmentations. OrganoID tracking results were compared to manually tracked images. The OrganolD platform and test microscopy images were distributed to an independent user to confirm reproducibility of all metrics, statistics, and figures. 
bioRxiv preprint doi: https://doi.org/10.1101/2022.01.13.476248; this version posted January 16, 2022. The copyright holder for this preprint (which was not certified by peer review) is the author/funder, who has granted bioRxiv a license to display the preprint in perpetuity. It is made available under aCC-BY-NC-ND 4.0 International license.

a

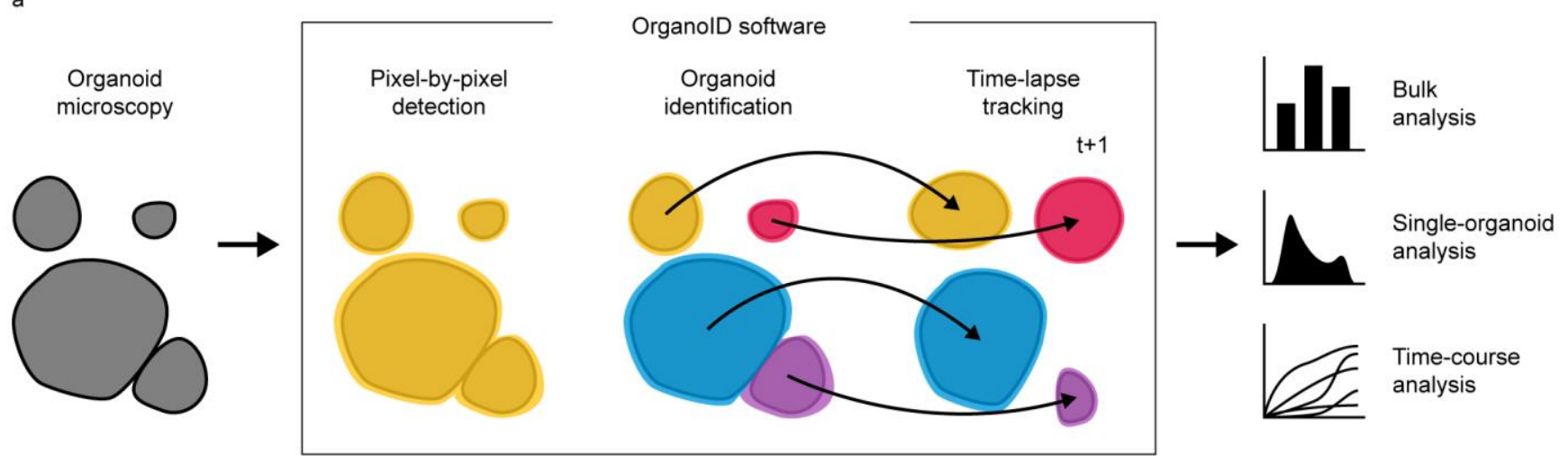

b

Image size $\left(\right.$ pixels $\left.{ }^{2}\right)$

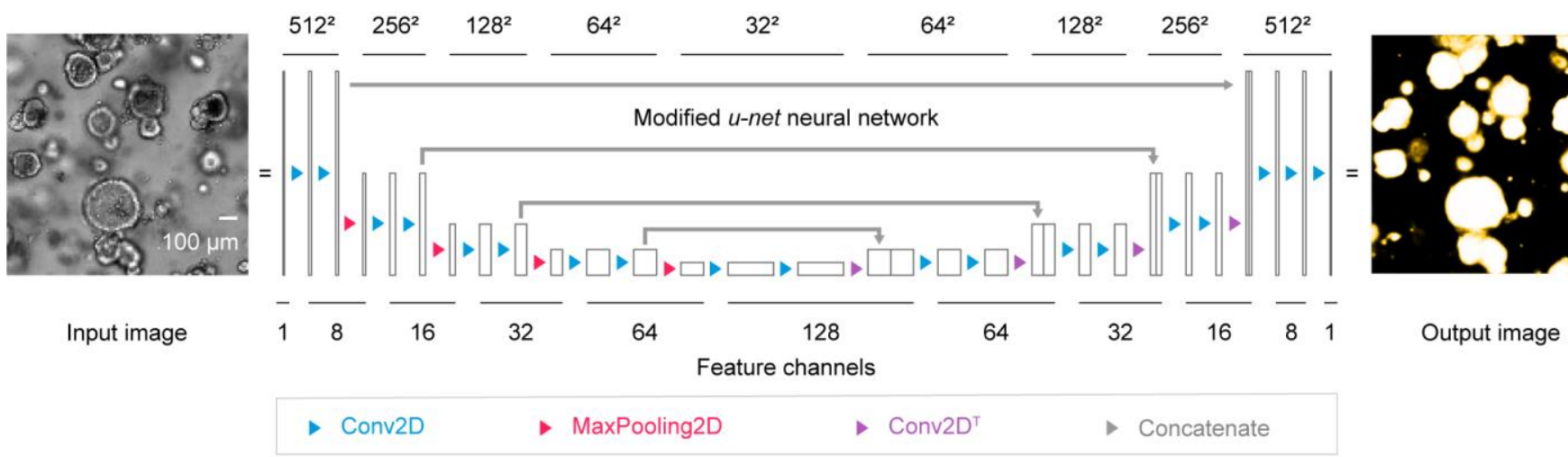

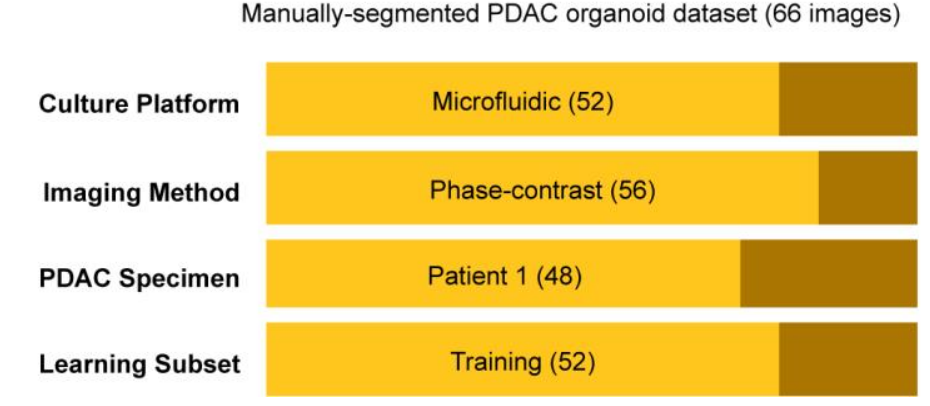

Well plate (14)

Brightfield (10)

Patient 2 (18)

Validation (14) d Augmented training dataset (2000 images)

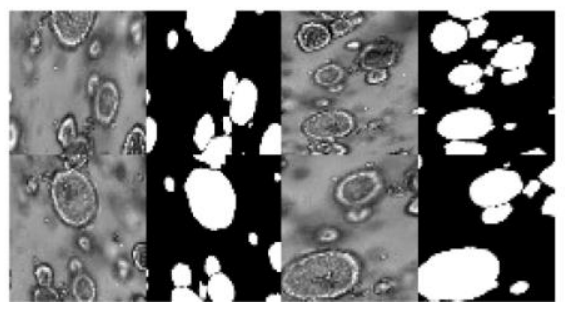

Figure 1. (a) The OrganolD software automates robust analysis of organoid microscopy images. Contours are detected pixel by pixel and then separated into distinct organoids for bulk or single-organoid analysis. Identified organoids can also be tracked across time-lapse image sequences to follow responses over time. (b) Microscopy images are processed by a convolutional neural network to produce images that represent the probability that an organoid is present at each pixel. The network follows the $u$-net architecture, which applies a series of twodimension convolutions, maximum filters, and image concatenations to extract and localize image features. Feature channel depths were minimized to limit overfitting and computational power required to use the tool. Scale bar $100 \mu \mathrm{m}$. (c) The network was trained on 66 manually labeled microscopy images of organoids derived from pancreatic ductal adenocarcinoma (PDAC) samples from two patients. Organoids were cultured in a well plate or microfluidic format and imaged through phase-contrast or brightfield microscopy. Images were then split into datasets for network training (80\%) and validation (20\%). (d) The 52 images in the training dataset were passed through a series of random transformations to produce an augmented dataset of 2,000 images. 
a

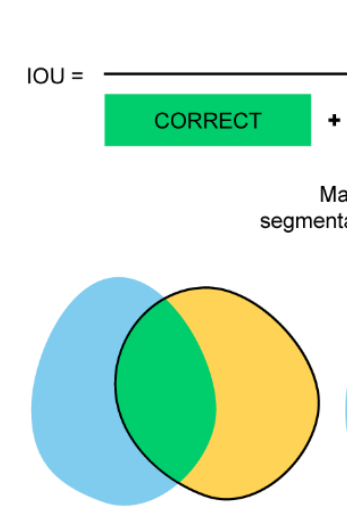

$\mathrm{IOU}=0.25$

b

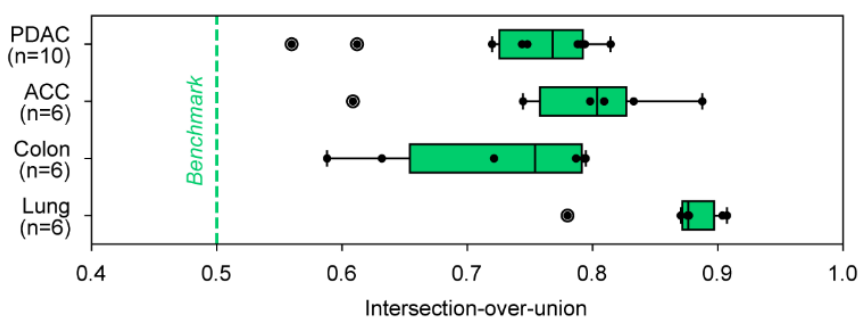

\section{CORRECT}

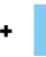

MISSED
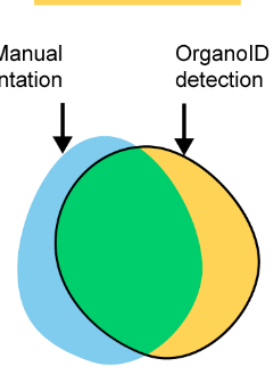

$\mathrm{IOU}=0.50$

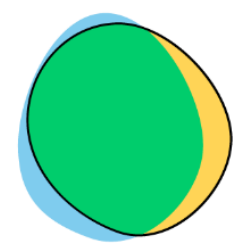

$\mathrm{IOU}=0.75$

\section{INCORRECT}

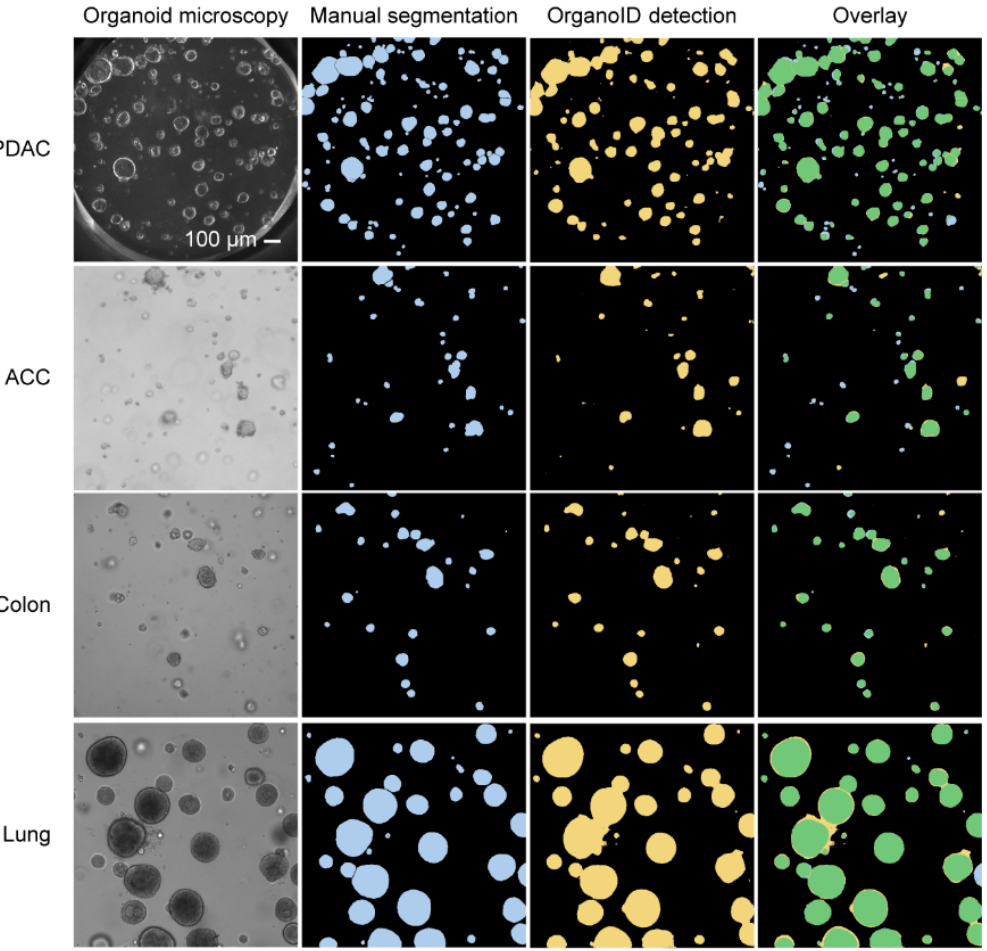

Figure 2. (a) The intersection-over-union (IOU) metric, defined as the ratio of the number true positive pixels to the union of all positive pixels, was used to measure the quality of the neural network detections. To compute the IOU, pixels above 0.5 in the network prediction image were marked as positives. Examples of IOU values for several degrees of overlap are shown. (b) A test set of images of organoids derived from PDAC, salivary adenoid cystic carcinoma (ACC), colon epithelia, and distal lung epithelia were manually segmented to assess network performance. An IOU of 0.5 was set as a benchmark for a successful network prediction (dashed green line). All images in the test set passed the benchmark, which demonstrates the capacity of the PDAC-trained network to generalize to other organoid types. (c) Representative examples of the test dataset are shown with the corresponding manually produced segmentation image and OrganolD thresholded output. Scale bar 100 $\mu \mathrm{m}$. 
bioRxiv preprint doi: https://doi.org/10.1101/2022.01.13.476248; this version posted January 16, 2022. The copyright holder for this preprint (which was not certified by peer review) is the author/funder, who has granted bioRxiv a license to display the preprint in perpetuity. It is made available under aCC-BY-NC-ND 4.0 International license.
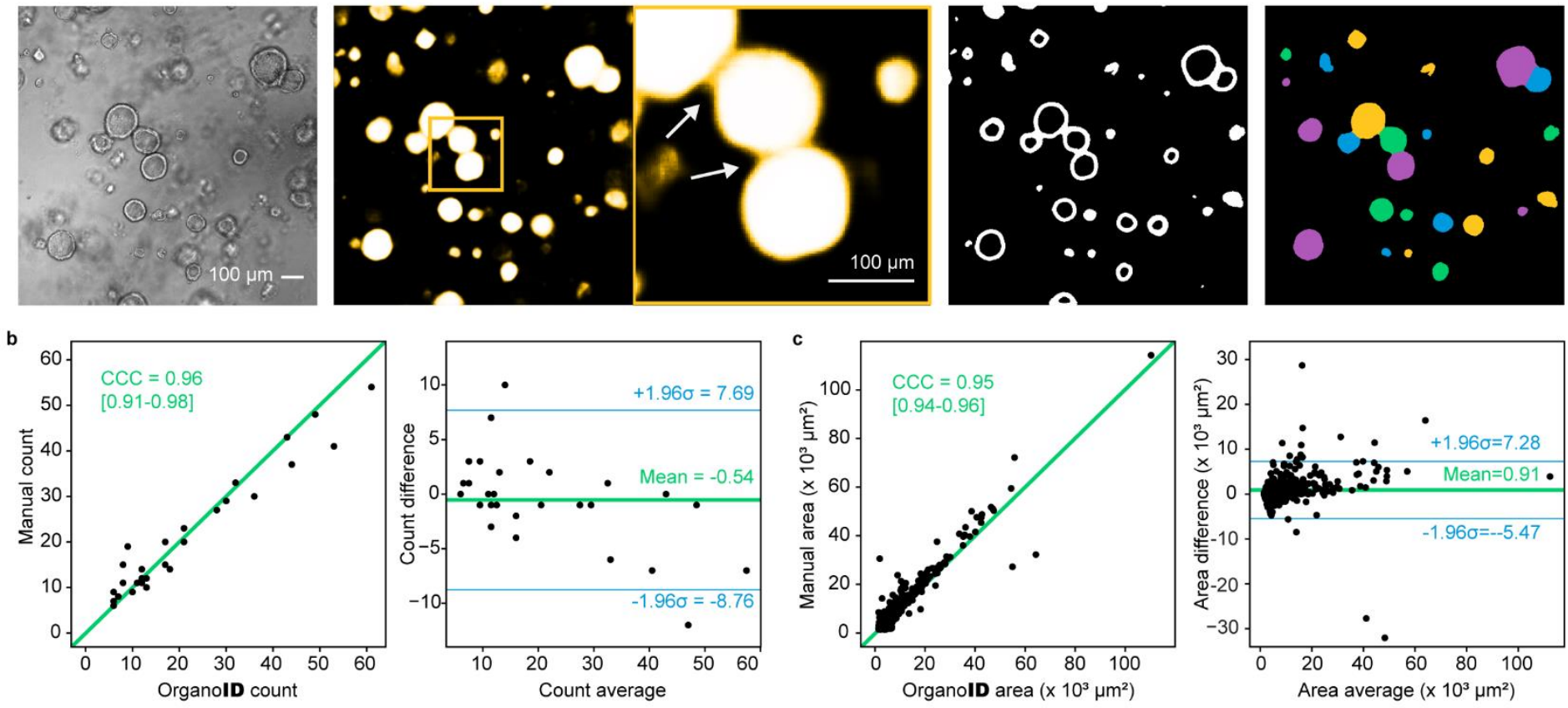

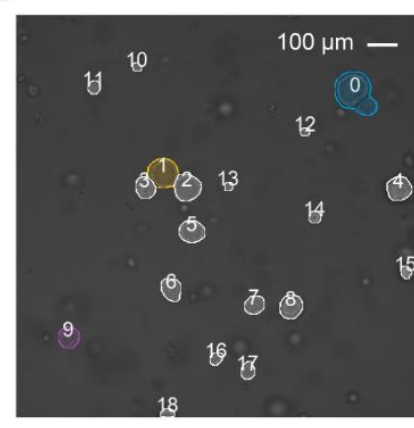

0 hours

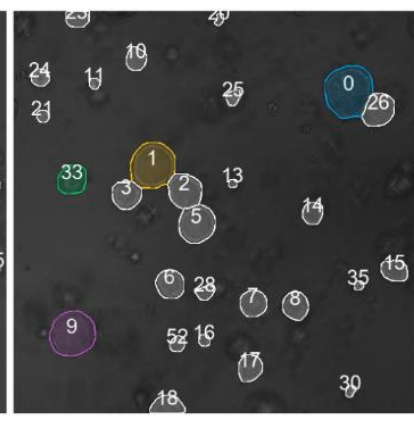

46 hours

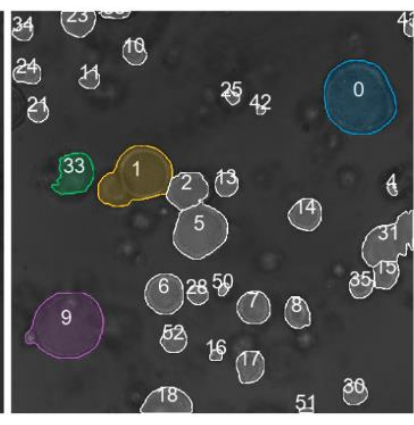

92 hours

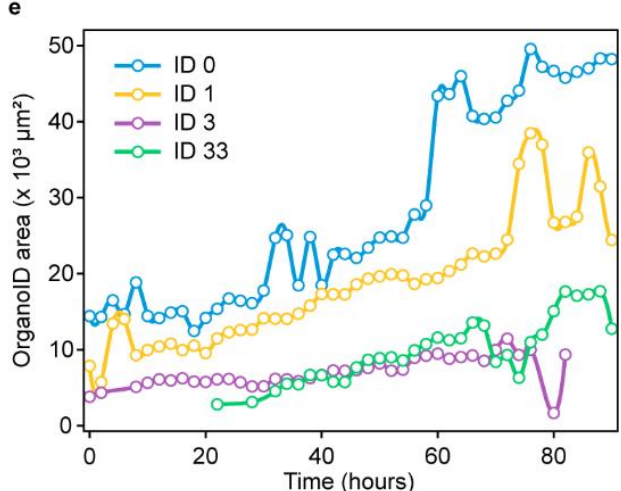

Figure 3. (a) OrganolD can identify individual organoid contours, including those in physical contact. An example image (left) is shown to demonstrate the steps of single-organoid identification. The neural network predictions (second-from-left) are observably less confident for pixels at organoid boundaries (enlarged view, indicated with white arrows), which enables edge detection with a Canny filter (second-from-right). Edges are used to identify organoid centers, which serve as basin initializers for a watershed transformation on the prediction image to produce a final single-organoid labeled image (right). (b) The identification pipeline was used to count the number of organoids in images from the test dataset. These counts were compared to the number of organoids in the corresponding manually segmented images. The concordance correlation coefficient (CCC) was computed to quantify measurement agreement (left). Bland-Altman analysis (right) demonstrates low measurement bias and limits of agreement. Black dots are test images. Green line in the left plot is $y=x$. (c) The area of each organoid in all test images was also measured manually and with OrganoID. Measurements were compared with CCC computation (left) and Bland-Altman analysis (right). Black dots are identified organoids. (d) Identified organoids in time-lapse microscopy images are matched across frames to generate single-organoid tracks and follow responses over time. Shown are images of three timepoints from an organoid culture experiment. (e) Automatically measured growth curves for a selected set of organoids from the experiment in (d). 

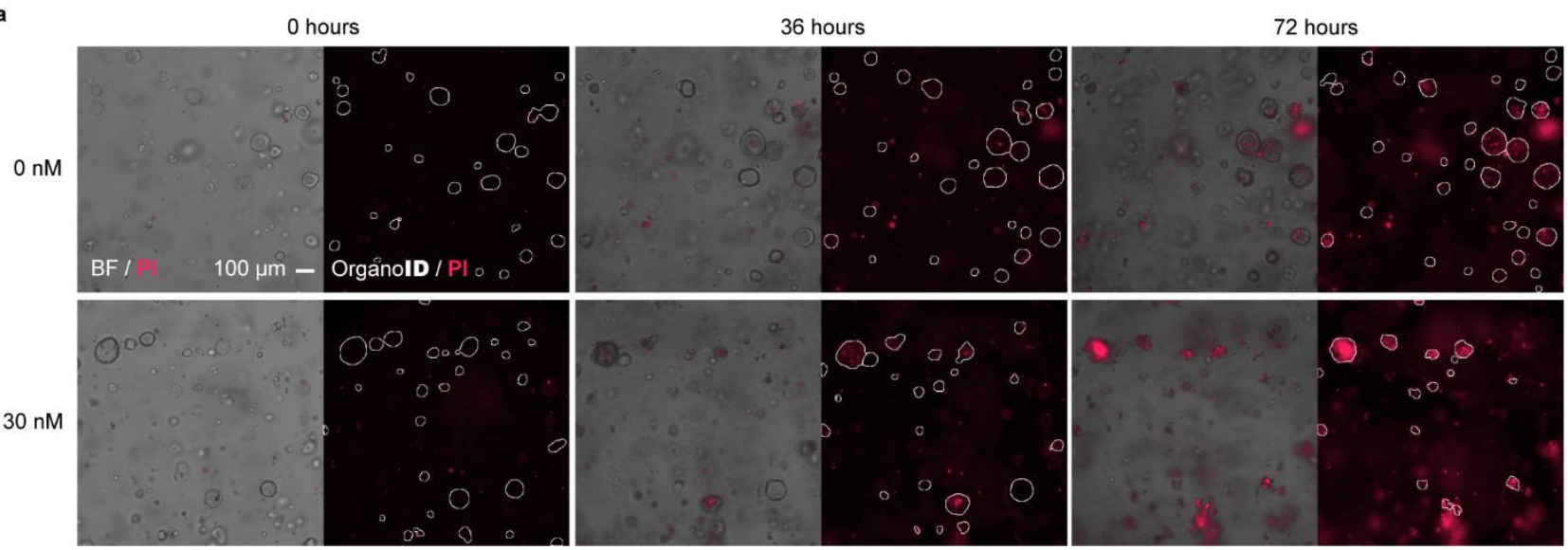

b

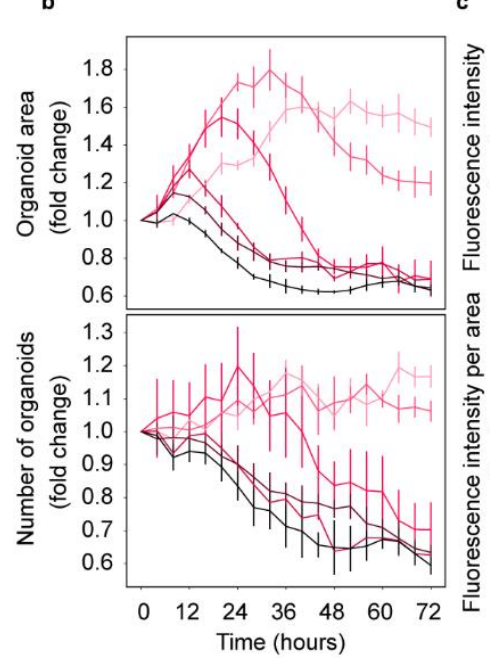

c

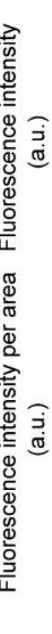

d

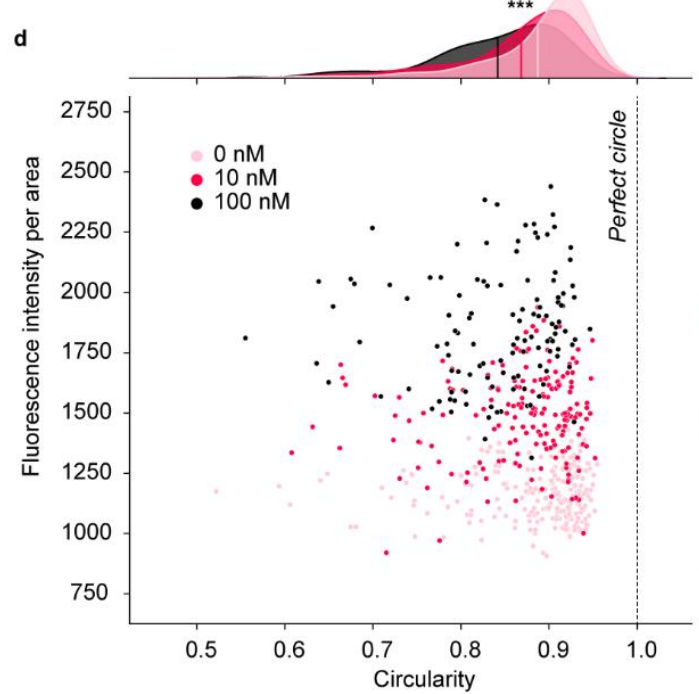

Figure 4. (a) PDAC organoids were treated with a serial dilution of gemcitabine ( $3 \mathrm{nM}$ to $1,000 \mathrm{nM})$ and imaged over 72 hours. Propidium iodide (PI) was used to fluorescently label dead organoids. Shown are representative brightfield images from three time points for control and $30 \mathrm{nM}$ gemcitabine conditions. OrganolD was used to identify organoid contours, which are displayed on top of the Pl fluorescence channel. (b) OrganolD measurements of total organoid area (top) and number of organoids (bottom) over time for each concentration of gemcitabine. Measurements were normalized to the initial timepoint. Error bars represent standard error of the mean $(n=6)$. (c) Total PI fluorescence intensity above control for each concentration of gemcitabine (top). Fluorescence intensity of OrganoID-identified regions were then summed and divided by the total organoid area (bottom), which improved separation of responses to different concentrations of gemcitabine. (d) Single-organoid measurements of fluorescence intensity, normalized to single-organoid area, and organoid circularity for three concentrations of gemcitabine at the 72-hour endpoint. Organoid circularity is defined as the ratio of the perimeter of a circle with the same area as the organoid to the actual perimeter of the organoid. Dots in the joint scatterplot are individual organoids. Marginal plots are kernel density estimates of the distribution of organoids for each concentration of gemcitabine. The central line for each distribution is the mean. ${ }^{* * *}: p<0.001$ for one-way ANOVA comparison of means across the three gemcitabine concentrations. 


\section{References:}

1. Kretzschmar, K. \& Clevers, H. Organoids: Modeling Development and the Stem Cell Niche in a Dish. Dev. Cell 38, 590-600 (2016).

2. Driehuis, E. et al. Oral Mucosal Organoids as a Potential Platform for Personalized Cancer Therapy. Cancer Discov. 9, 852-871 (2019).

3. Dutta, D., Heo, I. \& Clevers, H. Disease Modeling in Stem Cell-Derived 3D Organoid Systems. Trends Mol. Med. 23, 393-410 (2017).

4. Sachs, N. et al. A Living Biobank of Breast Cancer Organoids Captures Disease Heterogeneity. Cell 172, 373-386 (2018).

5. Clevers, H. \& Tuveson, D. A. Organoid Models for Cancer Research. Annu. Rev. Cancer Biol. 3, 223234 (2019).

6. Garreta, E. et al. Rethinking organoid technology through bioengineering. Nat. Mater. 20, 145-155 (2020).

7. Pettinato, G. et al. Spectroscopic label-free microscopy of changes in live cell chromatin and biochemical composition in transplantable organoids. Sci. Adv. 7, (2021).

8. Kim, S. et al. Comparison of Cell and Organoid-Level Analysis of Patient-Derived 3D Organoids to Evaluate Tumor Cell Growth Dynamics and Drug Response. SLAS Discov. 25, 744-754 (2020).

9. Dekkers, J. F. et al. High-resolution 3D imaging of fixed and cleared organoids. Nat. Protoc. 14, 17561771 (2019).

10. Hof, L. et al. Long-term live imaging and multiscale analysis identify heterogeneity and core principles of epithelial organoid morphogenesis. BMC Biol. 19, 1-22 (2021).

11. Bailey, S. R. \& Maus, M. V. Gene editing for immune cell therapies. Nat. Biotechnol. 37, 1425-1434 (2019).

12. Ang, L. T. et al. A Roadmap for Human Liver Differentiation from Pluripotent Stem Cells. Cell Rep. 22 , 2190-2205 (2018).

13. Riss, T., Trask, O. J. \& Jr. Factors to consider when interrogating 3D culture models with plate readers or automated microscopes. Vitr. Cell. Dev. Biol. 57, 238-256 (2021).

14. Borten, M. A., Bajikar, S. S., Sasaki, N., Clevers, H. \& Janes, K. A. Automated brightfield morphometry of 3D organoid populations by OrganoSeg. Sci. Rep. 8, 1-10 (2018). 

available under aCC-BY-NC-ND 4.0 International license.

15. Kassis, T., Hernandez-Gordillo, V., Langer, R. \& Griffith, L. G. OrgaQuant: Human Intestinal Organoid Localization and Quantification Using Deep Convolutional Neural Networks. Sci. Rep. 9, 1-7 (2019).

16. Kok, R. N. U. et al. OrganoidTracker: Efficient cell tracking using machine learning and manual error correction. PLoS One 15, e0240802 (2020).

17. Ronneberger, O., Fischer, P. \& Brox, T. U-net: Convolutional networks for biomedical image segmentation. in International Conference on Medical image computing and computer-assisted intervention 234-241 (Springer Verlag, 2015). doi:10.1007/978-3-319-24574-4_28

18. Clevert, D.-A., Unterthiner, T. \& Hochreiter, S. Fast and Accurate Deep Network Learning by Exponential Linear Units (ELUs). 4th Int. Conf. Learn. Represent. ICLR 2016 - Conf. Track Proc. (2015).

19. Schuster, B. et al. Automated microfluidic platform for dynamic and combinatorial drug screening of tumor organoids. Nat. Commun. 11, 1-12 (2020).

20. Canny, J. A Computational Approach to Edge Detection. IEEE Trans. Pattern Anal. Mach. Intell. (1986).

21. Munkres, J. Algorithms for the Assignment and Transportation Problems. J. Soc. Ind. Appl. Math. 5, 3238 (1957).

22. Romero-Calvo, I. et al. Human Organoids Share Structural and Genetic Features with Primary Pancreatic Adenocarcinoma Tumors. Mol. Cancer Res. 17, 70-83 (2018).

23. Fujii, M. et al. Human Intestinal Organoids Maintain Self-Renewal Capacity and Cellular Diversity in Niche-Inspired Culture Condition Human Intestinal Organoids Refined Condition IGF-1/FGF-2 Conventional Condition scRNA-seq. Stem Cell 23, 787-793.e6 (2018).

24. Sachs, N. et al. Long-term expanding human airway organoids for disease modeling. EMBO J. 38, e100300 (2019).

25. Bloice, M. D., Stocker, C. \& Holzinger, A. Augmentor: An Image Augmentation Library for Machine Learning. J. Open Source Softw. 2, 432 (2017).

26. Kingma, D. P. \& Ba, J. Adam: A Method for Stochastic Optimization. 3rd Int. Conf. Learn. Represent. ICLR 2015 - Conf. Track Proc. (2014).

27. He, K., Zhang, X., Ren, S. \& Sun, J. Delving Deep into Rectifiers: Surpassing Human-Level Performance on ImageNet Classification.

28. Virtanen, P. et al. SciPy 1.0: fundamental algorithms for scientific computing in Python. Nat. Methods $202017317,261-272(2020)$. 
bioRxiv preprint doi: https://doi.org/10.1101/2022.01.13.476248; this version posted January 16, 2022. The copyright holder for this preprint (which was not certified by peer review) is the author/funder, who has granted bioRxiv a license to display the preprint in perpetuity. It is made available under aCC-BY-NC-ND 4.0 International license.

29. Walt, S. van der et al. scikit-image: image processing in Python. PeerJ 2, e453 (2014).

30. Zhao, N., Powell, R.T., Yuan, X. et al. Morphological screening of mesenchymal mammary tumor organoids to identify drugs that reverse epithelial-mesenchymal transition. Nat Commun 12, 4262 (2021). https://doi.org/10.1038/s41467-021-24545-3 
10

te

0

13

13

(5)

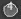

14

$t^{2}=0$

15

$x_{x}=$

8.

1913

18 